\title{
Editorial
}

\section{Ética del cuidado y robots}

\author{
Ética do cuidado e robôs
}

\section{Ethics of care and robots}

\author{
Carmen Domínguez-Alcón \\ PhD Sociology. École des Hautes Études en Sciences Sociales. París. Universidad de Barcelona. España. \\ Cómo citar esta editorial en edición digital: Domínguez-Alcón, C. (2017). Ética del cuidado y robots. \\ Cultura de los Cuidados (Edición digital), 21(47). \\ Recuperado de http://dx.doi.org/10.14198/cuid.2017.47.01 \\ CCorrespondencia: Emplear correo electrónico para cualquier comunicación con la autora. \\ Correo electrónico: cda.dominguez@ub.edu; Carmen.dominguez.alcon@gmail.com \\ Recibido: Autor invitado
}

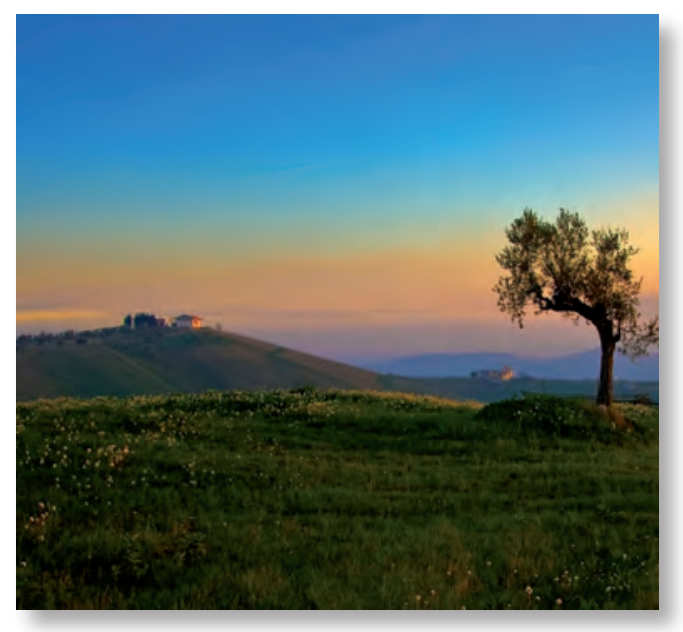

ABSTRACT

In this editorial, the author reflects on the incidence of robots in the nursing care and its ethical implications.

Keywords: nursing, robotics, care, technology and health.

\section{RESUMO}

Neste editorial, o autor reflete sobre o impacto de robôs nos cuidados enfemería e suas implicações éticas.

Palavras chave: enfermagem, robótica, cuidado, tecnologia e saúde.

\section{RESUMEN}

En esta editorial, se reflexiona sobre la incidencia de los robots en los cuidados de enfermería y sus implicaciones éticas.

Palabras clave: enfermería, robótica, cuidados, tecnología y salud.

¿Cuál es el papel de los robots en las prácticas de cuidado? ¿Qué sabemos acerca de cómo son aceptados por las personas cuidadas? ¿Son percibidos como colaboradores por las enfermeras? Estas y otras muchas cuestiones se plantean al pensar en robots y cuidados. Lo cierto es que la tecnología y la robótica están cambiando nuestras vidas, ambos términos nos sugieren máquinas complejas y múltiples dispositivos de uso habitual que "supuestamente" facilitan nuestra vida. Estamos familiarizados con los cambios que las tecnologías han ido introduciendo en distintos ámbitos: producción, conducción, transporte, logística; agentes inteligentes en el hogar, salud y asistencia $\mathrm{u}$ otros ámbitos. La tendencia es a incrementar el uso de la tecnología en entornos variados y en la vida cotidiana. Por ejemplo 
humanoides en funciones de asistente personal ${ }^{1}$, incluso algunos dispositivos que nos pueden parecer del futuro ya están funcionando en algún lugar. Lo que hasta hace poco parecía ciencia ficción está ya formando parte de proyectos realizados en algún laboratorio, es experimentado como innovación, o forma parte de prácticas avanzadas en algún sector.

La presencia de robots es frecuente en distintas aplicaciones, y en el entorno asistencial se debaten las posibilidades en el cuidado directo, mediante el estudio de la interacción humana con robots (HRI), y se muestra la importancia del papel de la cultura en esa compleja relación (Schwiebert 2013, p.8). En el ámbito del cuidado la información existente sobre el uso de la robótica evidencia el avance realizado. Se plantea a menudo la necesidad del uso de robots debido al incremento de personas mayores y muy mayores que precisan cuidados y pueden beneficiarse de los avances tecnológicos, aunque frente al tema las posturas son diversas, incluido el rechazo (Fitzpatric 2011). La oferta de robots existente es variada, y el deseo es armonizar las nuevas necesidades de cuidado con las posibilidades y funcionamiento de los robots. El objetivo es utilizar la ayuda eficaz que las aplicaciones de la robótica ofrecen, manteniendo la independencia de las personas usuarias, y su autonomía de decisión. Ya en la actualidad varios robots se publicitan como "robot enfermero". Por ejemplo Robear es un asistente para personas mayores con problemas de movilidad ${ }^{2}$. Riba II puede sustituir a enfermeros para coger en brazos a una persona que está en la cama y depositarla en una silla, o cogerla del suelo ${ }^{3}$. O Asimo que se considera el robot humanoide más avanzado.

Existen varios robots entre los denominados sociales, asistenciales, cuidadores, enfermeros, entre otras denominaciones, relacionados con prácticas de cuidado, que presentan diferentes características que podrían enunciarse como (1) Educativos y de entretenimiento, se trata de juguetes o dispositivos interactivos cuya finalidad es el aprendizaje, comunicarse, interactuar, entretener. (2) Terapéuticos, algunos con apariencia de peluche, se basan en la interacción animal. El objetivo es reducir el stress, estimular la interacción, y la socialización.(3) Asistentes personales inteligentes, basados en el sistema de reconocimiento de voz, que permiten pedir al móvil o a otro dispositivo que realice algunas funciones. (4) Robots cuidadores, que interactúan, y se comunican. El objetivo es ayudar a las personas mayores a vivir de forma independiente. (5) Robots médicos. (6) Robots cuyo uso se inició en la industria y actualmente tratan de adaptar sus aplicaciones a tareas asistenciales. Otro tipo son los robots sexuales, o de placer, o entretenimiento, de aspecto humanoide.

Conocer los distintos tipos de robots y dispositivos que ofrecen la posibilidad de mejorar algunas capacidades, ayuda a visualizar las diferencias que existen entre ellos, según las características que presentan, y la utilidad que pueden suponer en distintas situaciones. Algunos robots funcionan desde hace años y sus fabricantes han introducido mejoras en las sucesivas generaciones. Sin embargo, todavía sabemos poco de la percepción que cada uno

\footnotetext{
${ }^{1}$ Robots avanzados en 2017- https://www.youtube.com/watch? $v=z$ FAOeJ5NfB8 https://www.elandroidelibre.com/2016/12/plen-cube-robot-asistente-personal.html https://www.youtube.com/watch? $v=k b a D d g 4 L A 9 k$

${ }^{2}$ https://www.xataka.com/robotica-e-ia/robear-es-un-robot-oso-japones-enfermero-del-futuro)

${ }^{3}$ https://hipertextual.com/2011/08/riba-ii-robot-enfermero-japon
} 
de ellos puede despertar en diferentes grupos de población, e incluso entre los propios profesionales. Lo que hace necesario profundizar en esa vertiente. Desde el robot "personificado" animado, que promueve una interacción compleja, al robot "mecánico" que se utiliza como herramienta, que incluso en cirugía robótica la persona sobre la que actúa quizá no llega a verlo materialmente, hasta la variedad de dispositivos disponibles IoT y wearables ${ }^{4}$. Es obvio que cada sistema tiene su utilidad en cada contexto. Lo que nos lleva a algunas reflexiones acerca de la interacción entre humanos y robots desde la aplicación de la ética del cuidado en la práctica enfermera.

Desde que Carol Gilligan identificó las diferencias por razón de género en el razonamiento moral (Gilligan 1982), la ética del cuidado se ha desarrollado y se aplica de diferentes formas (Barnes y Brannelly 2008, p.384). Para la reflexión en el tema que propone el presente editorial se utiliza la aproximación de Joan Tronto que define el cuidado como la "actividad genérica que comprende todo lo que hacemos para mantener, perpetuar, reparar nuestro mundo de manera que podamos vivir en el lo mejor posible. Este mundo comprende nuestro cuerpo, nosotros mismos, nuestro entorno, y los elementos que buscamos enlazar en una red compleja de apoyo a la vida" (Fisher y Tronto 1990, 38). Esa definición, de 1990, es la misma que plantea posteriormente en Moral Boundaries (Tronto, 1994) obra en la que esa autora realiza un análisis pionero, sugerente y relevante, señalando la dimensión política del cuidado. Proponiendo cuatro fases analíticas y los aspectos morales vinculados a cada una de ellas, a las que posteriormente añade una quinta fase (Tronto 2013). Considera que en el cuidado es decisivo tener en cuenta las necesidades del otro/otros para guiar la acción. De manera que el cuidado implica una forma de relación activa (Domínguez Alcón et al. 2013, pp.91 ss. y 157 ss.).

Para comprender y concretar todas las dimensiones del cuidado Tronto plantea considerarlo como un proceso social continuo con distintas fases: La primera fase que denomina preocuparse de ("caring about") implica el reconocimiento de las necesidades y la evaluación de la posibilidad de aportar una respuesta. Es una preocupación que está matizada individual y culturalmente. Incluye también el nivel social y político. La segunda fase que corresponde a asumir la responsabilidad ("caring for") supone asumirla en relación con la necesidad identificada, así como determinar la naturaleza de la respuesta a aportar. La tercera fase realizar el cuidado ("care giving") es el trabajo material, tomando contacto directo con quien recibe el cuidado. En la cuarta fase que señala como recibir el cuidado/ servicio / actividad ("care receiving") se supone que el objeto de solicitud responde al cuidado que recibe. La autora pone énfasis en la importancia de incluir y considerar la recepción del cuidado entre los elementos del proceso, así como las preferencias de quien recibe el cuidado. Se trata de ofrecer una respuesta accesible, adaptada, y aceptable a la necesidad (Domínguez Alcón 1996, 1997). Es esencial que la respuesta sea accesible, ya que la existencia de respuesta no siempre supone que quien la precisa pueda acceder a ella. La idea de adaptabilidad es relevante, ya que la existencia de respuesta implica que sea apropiada a la necesidad que se preten-

\footnotetext{
${ }^{4}$ Internet de las cosas (IoT) y llevables (wearables) con expectativas remarcables de uso para el cuidado y de aplicabilidad en la practica enfermera. Y la tele asistencia.
} 
de cubrir. La inclusión de la aceptabilidad por parte de quien recibe el cuidado, es primordial ya que supone respetar a la otra persona como interlocutor, sujeto del cuidado, persona con dignidad, derechos, y capacidad de decidir, lejos de considerarla meramente objeto receptor del cuidado, servicio, o atención, sin capacidad de decisión, ni participación. La fase final (caring with") requiere que las necesidades de cuidado y las formas de satisfacerlas sean compatibles con los compromisos democráticos de justicia igualdad y libertad para todos (Tronto, 2013, p.23).

Proporcionar y recibir cuidado es una parte importante de la vida de las personas. La aproximación de Tronto coloca la ética del cuidado en un contexto moral y político, cuya puesta en práctica, a partir de las fases que la autora identifica, suponen un punto de partida para reflexionar sobre los elementos necesarios, en cuanto a aspectos morales y sociales vinculados a cada una de las fases que plantea. Así a la primera fase "preocuparse de" le corresponde un valor o virtud de la atención; a la segunda "asumir la responsabilidad" le corresponde el valor responsabilidad; a la tercera fase "realizar la actividad" le corresponde la competencia; y por último a la cuarta fase "recibir el cuidado" le corresponde la sensibilidad/ capacidad de respuesta. Tales elementos/ componentes/ valores/ virtudes citadas, son esenciales en relación con la intervención en políticas públicas, ya que pueden ser: (a) ignorados con las implicaciones que la no consideración pueda suponer; (b) institucionalizados y posibilitar el avance hacia bases más permanentes y positivas en la consideración y provisión del cuidado; y (c) dar lugar a estereotipos que marquen negativamente la construcción del objeto de intervención. Al añadir una quinta fase ("caring with") Tronto apunta como cua- lidades necesarias: pluralidad, comunicación, confianza, respeto, y solidaridad

Los aspectos de: atención, responsabilidad, competencia, capacidad de respuesta, y confianza se consideran los principios esenciales de la ética del cuidado. Los estudios realizados (Barnes y Brannelly 2008, p. 384) muestran como la ética del cuidado puede usarse como marco para analizar prácticas sociales y de cuidado, ya que los principios pueden servir de guía en diferentes formas de práctica, como es el caso que se plantea en la reflexión crítica acerca de los robots y las prácticas de cuidado. Para la práctica enfermera la utilización de alta tecnología es frecuente y supone una ayuda valiosa. En relación con los cuidados una cuestión esencial es preservar el protagonismo y participación de la persona que necesita utilizar cualquier dispositivo tecnológico. Cada contexto requiere tener en cuenta condiciones y particularidades específicas. Al utilizar la ayuda eficaz que las aplicaciones de la robótica ofrecen, la preocupación es mantener la independencia de las personas usuarias, y su autonomía de decisión.

El objetivo final es proveer "buen cuidado" y el enfoque es "hacer con", "estar con". En la perspectiva que supera el "hacer para" de períodos anteriores, que dejan al margen a la persona sujeto del cuidado, ya sea -el cuidadorealizado otra persona, profesional, enfermera, cuidadora, o que se realiza mediante la ayuda de robots o de otros dispositivos técnicos facilitadores. El buen cuidado en la perspectiva que defiende Gastmans (2012) supone: "respetar a la persona; obtener su consentimiento; proteger la información confidencial; cooperar con los demás profesionales; mantener la competencia profesional, y minimizar los riesgos". Para Barnes y Brannelly (2008, p.386) el buen cuidado requiere de una continua negociación entre 
quienes proveen el cuidado y quienes lo reciben. Desarrollar prácticas basadas en los principios de la ética del cuidado tiene el potencial de permitir un dialogo que incluye las perspectivas de los actores implicados en la situación de cuidado y proporciona un lenguaje común.

Las nuevas direcciones del cuidado se relacionan con lo que es significativo para las personas en cada situación de vida que precisa cuidado. El contexto y las condiciones en que se desarrolla el cuidado muestran los elementos esenciales en los que la práctica enfermera centra la atención. Giran alrededor de acompañar, orientar, enseñar, facilitar, tomar decisiones, aplicar con competencia e inteligencia los complejos conocimientos propios de la disciplina, utilizar la tecnología para mejorar la calidad de vida y del cuidado. Tienen en cuenta las raíces culturales, las creencias, lo que es significativo para las personas implicadas en las situaciones de vida que precisan cuidado. El objetivo es el buen cuidado, aplicar con inteligencia los saberes y el conocimiento profesional, restaurar la fuerza, el deseo de vivir, acompañar y facilitar el bienestar en cualquier situación, con preocupación, solicitud, con respeto a la dignidad y autonomía de todas las personas.

De manera que en la reflexión acerca del uso de robots en el cuidado de las personas, parece razonable la propuesta de utilizar como guía los principios esenciales de la ética del cuidado. Además de ampliar el conocimiento de las tecnologías y los robots en la practica enfermera. Tarea a la que ya se han aplicado las enfermeras (Wilson 2014; ANA, 2014; Mieronkoski, Azimi, Rahmani et al. 2017) y con ello aportar elementos para el debate acerca de un tema controvertido pero imprescindible. Que también es objeto de preocupación e interés a nivel del Parlamento Europeo que ha publicado un informe (Delvaux, 2016) con varias recomendaciones destinadas a la Comisión sobre normas de Derecho civil sobre robótica. Preocupación que en los últimos años (Sharkey 2008) se viene expresando desde diferentes instancias

\section{Bibliografía}

- American Nurses Association (2015). Wearable Technology for Nurses. American Nurses Association. Recuperado de http://www.nursingworld.org/Wearable-Technology-for-Nurses

- Barnes, M., \& Tula, B. (2008). Achieving Care and Social Justice for People with Dementia. Nursing Ethics, 15 (3), 384-395.

- Delvaux, M. (2016). Proyecto de Informe con Recomendaciones destinadas a la Comisión sobre normas de Derecho civil sobre robótica 2015/2103(INL). Bruselas: Parlamento Europeo. Comisión de Asuntos Jurídicos.

- Domínguez Alcón, C. (1997). Familia, cuidados informales y políticas de vejez pp. 464-479 en A. S. Staab y L.C. Hodges Enfermería gerontológica. México: McGraw Hill.

- Domínguez-Alcón, C.et al.(2013). Qué políticas para qué igualdad Valencia: Tirant lo Blanc.

- Domínguez-Alcón, C. (2017). Evolución del cuidado y profesión enfermera Barcelona: Ediciones San Juan de Dios..

- Fisher, B. \& Tronto, J. (1990). Toward a Feminist Theory of Caring pp. 35-61 en Emily Abel y Margaret Nelson Circles of Care. Nueva York: University of New York Press.

- Fitzpatric, M. (2011). No robot: Japan's elderly fail to welcome their robot overloads. BBC News 4, febrero.

- Gastmans, C. (2012). Dignity-Enhancing Care for Persons with Dementia and its Application to Advance Euthanasia Directives. en: Y. Denier, C. Gastmans, A. Vandevelde (Eds.) Justice, Luck \& Responsibility. Philosophical Background and Ethical Implications for End-ofLife Care. Dordrecht: Springer.

- Gilligan, C. (1982). In a Different Voice. Cambridge: Harvard University Press.

- Mieronkoski, R., Azimi, I., Rahmani, A.M.,, Aantaa, R., Terävä, V., Liljeberg, P., Salanterä, S. (2017). The Internet of Things for basic nursing care-A scoping review" International Journal of Nursing Studies 69 (2017) 78-90.

- Sharkey, N. (2008). The Ethical Frontiers of Robotics. Science 322(5909), 1800-1801.

- Schwiebert, A. (2013). Could a nurse robot cry? Nurses 'perspectives on medical robots. Michigan: University of Michigan.

- Tronto, J. (1994). Moral Boundaries. Nueva York: Rouledge.

- Tronto, J. (2013). Caring Democracy: Markets, Equality and Justice. Nueva York: Nueva York University Press.

- Wilson, B. (2014). The Nerdy Nurse's Guide to Using Technology, Indianapolis: Sygma Theta Tau International. 